\title{
Rheumatoid arthritis and robotic radical surgery: Positioning and anaesthetic challenges.
}

\author{
U Hariharan $^{I^{*}}$, A Kulkarni ${ }^{2}$, AK Mittal $^{3}$, AK Bhargava $^{4}$ \\ Fellow Oncoanaesthesia ${ }^{1}$, Senior Consultant Anaesthetist ${ }^{2}$, Consultant Anaesthetist ${ }^{3}$, Director and Head \\ Anaesthesia ${ }^{4}$, Rajiv Gandhi Cancer Institute and Research Centre, Sector 5, Rohini, Delhi 85, India.
}

*Corresponding author: uma1708@gmail.com

\begin{abstract}
Robotic surgery is the future of minimally invasive surgery. Anaesthesiologists have to be prepared for the various challenges posed by robotic surgeries in terms of steep trendelenberg position, prolonged pneumoperitoneum and fluid restriction. Rheumatoid arthritis (RA) is a debilitating autoimmune inflammatory arthritis affecting almost every joint of the body. The various deformities due to RA can pose a challenge for proper positioning. It can also cause difficult venous cannulation, difficult airway, impaired respiratory function, multi-system involvement and propensity to cause neurovascular impingement. We present a case of severe rheumatoid arthritis with hypothyroidism posted for elective robotic radical hysterectomy and the anaesthetic challenges.
\end{abstract}

Keywords: Rheumatoid arthritis, robotic surgery, radical hysterectomy, steep trendelenberg, positioning

\section{Introduction}

Robotic surgery is a new feather in the revolutionary cap of minimally invasive surgery. Anaesthesiologists have to be prepared for the various challenges posed by different robotic surgeries in terms of steep trendelenberg position, prolonged pneumoperitoneum and fluid therapy. Rheumatoid arthritis (RA) is a debilitating autoimmune inflammatory arthritis causing joint destruction and multi-system involvement. The various deformities due to RA can cause difficulty in positioning, venous access, difficult airway, impairment of pulmonary function and propensity to cause neurovascular impingement. The advantages of robotic surgery over conventional can be particularly beneficial in such high risk patients.

\section{Case Report}

A 42 year old female patient with severe RA (bed-ridden for the past 6 months) was posted for robotic radical hysterectomy with bilateral salpingo-oopherectomy, pelvic lymph node dissection, omental biopsy and retroperitoneal node sampling for endometrial adenocarcinoma. She had typical dinner fork deformity of hands, fixed flexion deformity of elbows, hip and knee joints, with impossible shoulder or hip abduction. Her right lower limb was always over her left leg, causing pressure symptoms over points of contact. On airway assessment, mouth opening was 2 fingers; limited neck extension; irregular dentition (malocclusion) with upper incisor buck teeth and mobile lower incisors. She had compromised respiratory function (mild restrictive pattern in pulmonary function tests). Cardiac echocardiography showed no regional wall motion abnormality, ejection fraction $55 \%$, left ventricular hypertrophy, mild diastolic dysfunction and no evidence of pulmonary hypertension. Her current effort tolerance was poor due to immobility caused by RA. The patient was a known hypothyroid on thyroxine therapy. Her investigations were within normal limits, including thyroid function tests. She had already taken a course of disease modifying agents (methotrexate and acetylsalicylic acid) 2 years back for RA and was currently on non-steroidal antiinflammatory agents (NSAID) and intermittent oral prednisolone for inflammatory exacerbations.

Obtaining an intravenous access was a challenge. Invasive monitoring was planned. 
Arterial line was inserted in right dorsalis pedis (as both wrists were deformed and movements painful). Ultrasound-guided right internal jugular venous cannulation was done for central venous pressure monitoring, preinduction, under local anaesthesia. In view of anticipated difficult airway, C-MAC ${ }^{\mathrm{TM}}$ videolaryngoscope was used for intubation, after intravenous induction of anaesthesia with midazolam, fentanyl and propofol. Muscle relaxant was given after confirming correct tube placement, followed by continuous relaxant infusion (atracurium). Neuromuscular monitoring was employed to prevent even slight movement, which can be devastating after docking of robot. Maintenance of anaesthesia was with $\mathrm{O}_{2}+$ air + desflurane+ propofol infusion. Side docking of robotic instruments was done as hip abduction and lithotomy position was not possible. After supra-umblical primary trocar insertion, secondary robotic ports were inserted under vision, to prevent damage to viscera or vessels. Care was taken not to move any joint or body part forcefully. Patient was positioned in the same manner in which she was used to lying down before induction of anesthesia with maintenance of her natural body lie, to prevent inadvertent joint dislocation or musculoskeletal injury. All extremities were rolled in soft cotton bandages, including eye padding. Active measures were taken to prevent hypothermia. Since docking was done in an atypical manner, the operative time was prolonged by 30 minutes with minimal blood loss. Postsurgery, de-docking was done, pneumoperitoneum released after supine positioning. Following return of train-of-four response on neuromuscular monitor and spontaneous respiration, the patient was reversed but not extubated, in view of inadequate tidal volume. Analgesia was administered with intravenous paracetamol $(1 \mathrm{gm})$, port-site local anaesthetic infiltration and continuous intravenous fentanyl infusion (30- $40 \mathrm{mcg} / \mathrm{hr}$ ). She was shifted to the oncosurgical intensive care unit, put on pressure support ventilation initially, followed by CPAP (continuous positive airway pressure) mode and extubated over a tube exchanger device (due to known difficult airway) after 4 hours. Postoperatively, the patient maintained all vital parameters and was later transferred to the ward.

\section{Discussion}

This case highlights the paramount importance of gentle handling and positioning of such patients for complex surgeries like the robotic surgery. $\mathrm{RA}^{1}$ is an autoimmune disease causing pain, swelling, stiffness and loss of function of the involved joints. It also affects the laryngeal cartilages ${ }^{2}$ and cervical spine, predisposing to difficult airway. Robotic surgery is beneficial in such patients in view of less blood loss, minimal postoperative pain and better patient recovery. Padding of pressure points ${ }^{3}$, cushioning of bony prominences and maintaining the natural position of the deformed joints is mandatory. Steep trendelenberg $\left(40^{\circ}\right)$ position $^{4}$ during robotic surgery can lead to increase in central venous pressure, rise in intracranial and airway pressures, with impairment of respiratory function.

Positioning for robotic pelvic surgeries involve hip abduction, lithotomy, steep trendelenberg position, arms completely adducted and by the side of the patient. Patient falling is prevented by using appropriate shoulder supports and padded straps. The robot usually comes in between the two legs and the various ports/robotic arms are inserted to reach the pelvis. All these were difficult in our patient in view of severe RA. Modifications are required in anaesthesia and surgical technique according to the deformities in an individual patient.

In-view of anticipated difficult airway ${ }^{5}$, videolaryngoscope was used and muscle relaxant was given only after checking correct tube placement. Special precautions were taken to maintain adequate renal function, normocarbia and normothermia. She was put on short-term elective ventilation, due to pre-existing pulmonary dysfunction ${ }^{6}$, till return of normal respiratory function. Cautious extubation was done over a tube exchanger device ${ }^{7}$. Adequate 
pain management is imperative for better recovery in R.A patients.

\section{Conclusions}

Robotic surgery (the da Vinci ${ }^{\mathrm{TM}}$ surgical system) gives three-dimensional visualization and its instruments have 7 degrees of motion to mimic human dexterity ${ }^{8}$. It can be safely performed in RA patients, provided specific precautions are strictly followed. Anaesthetic considerations include limited access to patient, longer surgical times, prolonged pneumoperitoneum, changes due to steep trendelenberg position, fluid balance, good intraoperative muscle relaxation, safe positioning, prevention of neurovascular injury, maintenance of normothermia and postoperative pain relief ${ }^{9}$. Videolaryngoscope $\mathrm{e}^{10}$ is a standard tool in the management of difficult airway, where intubation can be done under vision in neutral head position. All operating room staff must be proficient in quick disengagement and dedocking of robotic instruments in the event of an emergency situation.

\section{References:}

1. Harris ED. Clinical features of rheumatoid arthritis. In: Ruddy S, Harris E, Sledge C (eds): Kelly's Textbook of Rheumatology. 6th ed. Philadelphia: WB Saunders, 2001, 9671000.

PMid:11496908

2. Bridger MW, Jahn AF, Van Nostrand AW: Laryngeal rheumatoid arthritis. Laryngoscope 1980;90:296-303. http://dx.doi.org/10.1288/00005537$\underline{198002000-00015}$

PMid:7354699

3. David JWK, Ravi PM. Patient positioning in anaesthesia. Continuing Education in Anaesthesia, Critical care and Pain 2004;4(5):160-63. http://dx.doi.org/10.1093/bjaceaccp/mkh044

4. Alain FK, Andre M, De Wolf, Jan FAH. Anesthetic considerations for robotic surgery in the steep trendelenburg position. Advances in Anesthesia 2012;30:75-96. http://dx.doi.org/10.1016/j.aan.2012.07.003

5. Jeffery LA, Carin AH, Robert AC. Practice guidelines for management of the difficult airway: An Updated Report by the American Society of Anesthesiologists Task Force on
Management of the Difficult Airway. Anesthesiology 2013;118(2):1-20.

6. Anaya JM, Diethelm L, Ortiz LA, Gutierrez M, Citera G, Welsh RA, et al. Pulmonary involvement in rheumatoid arthritis. Semin Arthritis Rheum. 1995 Feb;24(4):242-54. http://dx.doi.org/10.1016/S00490172(95)80034-4

7. Moyers G. Use of the Cooks Airway Exchange catheter in "bridging" the potentially difficult extubation: A case report. ANAA 2002;70(4):275-78.

8. Anthony RL, Andres EC, William CM. Robotic Surgery: A Current Perspective. Ann Surg 2004;239(1):14-21. http://dx.doi.org/10.1097/01.sla.0000103020.1 $9595.7 \mathrm{~d}$

PMid:14685095 PMCid:PMC1356187

9. Fengling J, Frances C. Multimodal analgesia for postoperative pain control. JCA 2001;13(7):524-539.

10. Aziz MF, Dillman D, Fu R, Brambrink AM. Comparative effectiveness of the C-MAC video laryngoscope versus direct laryngoscopy in the setting of the predicted difficult airway. Anesthesiology 2012;116(3):629-36. http://dx.doi.org/10.1097/ALN.0b013e318246 ea34

PMid:22261795 